\title{
Cloning and expression of a novel CREB mRNA splice variant in human testis
}

\author{
Xiaoyan Huang ${ }^{1}$, Jun Zhang ${ }^{1,2}$, Li Lu ${ }^{1}$, Lanlan Yin ${ }^{1}$, Min $\mathrm{Xu}^{1}$, Youqun Wang ${ }^{2}$, Zuomin Zhou ${ }^{1}$ \\ and Jiahao Sha ${ }^{1}$ \\ ${ }^{1}$ Key Laboratory of Reproductive Medicine, Nanjing Medical University, Nanjing, 210029, P.R. China \\ and ${ }^{2}$ Division of Pharmacology Research, China Pharmaceutical University, Nanjing, 210009, P.R. China
}

Correspondence should be addressed to Jiahao Sha; Email: shajh@njmu.edu.cn

\begin{abstract}
Identification of genes specifically expressed in adult and fetal testis is important in furthering our understanding of testis development and function. In this study, a novel human transcript, designated human testis cAMP-responsive element-binding protein (htCREB), was identified by hybridization of adult and fetal human testis CDNA probes with a human cDNA microarray containing 9216 clones. The htCREB transcript (GenBank Accession no. AY347527) was expressed at 2.35-fold higher levels in adult human testes than in fetal testes. Sequence and ntBLAST analyses against the human genome database indicated that htCREB was a novel splice variant of human CREB. RT-PCR-based tissue distribution experiments demonstrated that the htCREB transcript was highly expressed in adult human testis and in healthy sperm, but not in testes from patients with Sertoli cell-only syndrome. Taken together, these results suggest that the htCREB transcript is chiefly expressed in germ cells and is most likely involved in spermatogenesis.

Reproduction (2004) 128 775-782
\end{abstract}

\section{Introduction}

Spermatogenesis is a unique physiological process during which male germ cells undergo a series of differentiation steps leading to the production of mature haploid sperm cells. This process involves the coordinated expression of many genes with unique cellular and temporal specificities. One of the molecular mechanisms playing a major role in spermatogenesis is the CAMP-dependent signaling pathway, which involves modulation of a multigene family of transcription factors that contain the basic transactivation and DNA-binding domains (bZIP), such as cAMP-responsive element-binding protein (CREB), cAMPresponsive element modulator (CREM), and activating transcription factor-1 (ATF-1) (Habener et al. 1995, Scobey et al. 2001). CREB is a member of the bZIP family of transcription factors, which consists of two functionally distinct domains: a carboxyl-terminal dimerization and DNA-binding (bZIP) domain, and an amino-terminal transcriptional transactivation domain (Meyer and Habener, 1993, Andrisani 1999). Transactivation of gene transcription by CREB is dependent on the phosphorylation of a single serine within the phosphorylation domain ( $P$ box or kinase-inducible domain (KID)) by cAMP-dependent protein kinase A (PKA) (Gonzalez \&
Montminy 1989). CREB is expressed in nearly all tissues tested thus far. However, in the testis, investigators have identified a number of novel alternative exon splicings that result in the synthesis of mRNAs encoding multiple isoforms of CREB (Hoeffler et al. 1990, Ruppert et al. 1992, Waeber et al. 1993). In the rat testis, these alternatively spliced CREB mRNAs are spermatogenic, cycledependent and expressed during development of the germ and Sertoli cells (Daniel \& Habener 1998), indicating that the CREB isoforms may be major players in spermatogenesis.

Previous CDNA microarray hybridization studies in our laboratory identified a number of known genes as being alternatively spliced in human testis, including reticulon, HsMCAK, DEAD-box protein and RAD23B; some of these spliceoforms were demonstrated to be spermatogenesisrelated (Cheng et al. 2002, Yin et al. 2002, Zhou et al. 2002, Huang et al. 2004). In this paper, we report a novel spliceoform of CREB, designated htCREB (human testis CAMP-responsive element-binding protein), which was primarily expressed in human testis and was almost undetectable in testis samples from patients with Sertoli cell-only syndrome (there was a very weak band in patients 4 and 5). As compared with other spliceoforms of CREB, htCREB employs both alternative exons and 
alternative splice site selection, with the resulting transcript lacking the basic transactivating region-associated Q2 domain. Taken together, these results suggest that htCREB is likely to inhibit transcription and may play an important role in human spermatogenesis.

\section{Materials and Methods}

\section{Samples}

Informed consent was received from either the participants or their kin, and the ethics committee of Nanjing Medical University (China) granted research approval prior to sample collection. Human testis from adult men (37 years old) and elderly (73 years old) men were obtained from the Body Donor Center (Nanjing Medical University), and fetal testes were obtained from accidentally aborted (as in road accidents) 6-month-old fetuses (Clinical Reproductive Center, Nanjing Medical University). Testis tissue samples from five individuals with Sertoli cell-only syndrome (SCOS) were acquired via biopsy, and healthy volunteers with proven fertility and normal semen quality (assessed by WHO criteria, 1999) donated ejaculated sperm. Macroscopic and histologic examinations showed there was every level of spermatogenic cells and Sertoli cells around seminiferous tubules in adult human testis and elderly testis; however, there were fewer spermatid and spermatozoa in elderly testis than in adult testis. Moreover, in fetal testis, only Sertoli cells and prospermatogonia converged at the mediastinum.

\section{Construction of human cDNA microarray}

A total of 9216 positive phage clones were randomly picked from a human testis insert $\lambda$ phage cDNA library (Clontech, HI5503U) and PCR amplified. The resultant PCR products were spotted on nylon membranes to generate a human testis cDNA microarray. The cDNA microarray preparation and hybridization signal analyses were performed as previously described (Sha et al. 2002).

\section{Array scanning and analyzing clones of interest}

Fetal testis, adult testis and human spermatozoa probes were prepared by incorporation of ${ }^{33} \mathrm{P}$-labeled dATP in a reverse transcription reaction, using $2 \mu \mathrm{g}$ purified mRNA as the template. The arrays were scanned, and the radioactive intensity of each spot was linearly scanned with a 65536 gray-grade in a pixel of $50 \mu \mathrm{m}$ and read out with array gauge software (Fuji Photo Film, Tokyo, Japan). After subtraction of the background from an area where no PCR product was spotted, clones with intensities over 10 were considered positive, and were picked, sequenced and analyzed as described (Sha et al. 2002, Wang et al. 2004). The generated sequences were subjected to BLAST analysis (www.ncbi.nlm.nih.gov), which identified one as htCREB, a novel human CREB mRNA splice variant in human testis (GenBank Accession no. AY347527). Further
GenBank sequence analyses were performed to determine the htCREB homologs and their chromosomal localization, and the nucleic and deduced amino-acid sequences of htCREB were analyzed with Gene Runner software (www.generunner.com) and SMART PROGRAM (Schultz et al. 1998).

\section{Expression of the htCREB transcript in human testis and sperm}

To determine expression of htCREB in various developmental stages of testis, htCREB-specific RT-PCR was carried out on cDNA from adult (age 37 years) testes, ejaculated sperm, old (age 73 years) testes and fetal testes, together with testes with SCOS. RNA was extracted from the various sources with Trizol reagent (Gibco BRL, Grand Island, NY, USA) and reverse-transcribed into cDNA with AMV reverse transcriptase (Promega). The various cDNAs were PCR amplified with htCREB-specific primers (P1, 5' CCA GCC ATC AGT TAT TCA G3' (forward, nt 262-280, located in exon $\mathrm{C}$ of htCREB); P2, 5' AGA CTT CAG CAC TTC CTA C3' (reverse, nt 519-537, located between exons $\mathrm{F}$ and $\mathrm{H}$ )) (Fig. 3) in $20 \mu \mathrm{l} \mathrm{PCR}$ reactions containing $10 \times$ PCR buffer $(2 \mu \mathrm{l}), 25 \mathrm{mmol} / \mathrm{I} \mathrm{Mg}^{2+}(1.5 \mu \mathrm{l}), 2 \mathrm{mmol} / \mathrm{l}$ dNTPs $(1.5 \mu \mathrm{l})$, Taq DNA polymerase $(5 \mathrm{U} / \mu \mathrm{l})(0.1 \mu \mathrm{l})$, distilled water $(10.9 \mu \mathrm{l}), 5 \mathrm{pmol}$ primer $(1 \mu \mathrm{l})$, and template cDNA $(2 \mu \mathrm{l})$. The amplification conditions consisted of an initial denaturation at $94^{\circ} \mathrm{C}$ for $5 \mathrm{~min}$, followed by 30 cycles of $94^{\circ} \mathrm{C}$ for $30 \mathrm{~s}, 52^{\circ} \mathrm{C}$ for $30 \mathrm{~s}$ and $72{ }^{\circ} \mathrm{C}$ for $30 \mathrm{~s}$, with a final extension at $72{ }^{\circ} \mathrm{C}$ for $7 \mathrm{~min}$. The PCR products were analyzed with $1.5 \%(\mathrm{w} / \mathrm{v})$ agarose electrophoresis, and $\beta$-actin was amplified as the control. The PCR product of htCREB in adult testes was gel purified with a kit (QIAprep Spin Miniprep Kit; Qiagen) and cloned by the TA cloning system (Promega). The inserts were sequenced from one end by an automated DNA sequencer-ABI100 (model no. 377).

\section{Tissue distribution of the htCREB transcript}

For determination of htCREB tissue distribution, cDNAs from 16 different human tissues (heart, brain, placenta, lung, liver, skeletal muscle, kidney, pancreas, spleen, thymus, prostate, testis, ovary, small intestine, colon and leukocytes) from a commercial human multiple tissue cDNA panel (MTC Panels I and II, cat. nos. K1420-1 and K1421-1; Clontech) were amplified and analyzed as described above, with $\beta$-actin as the positive control.

\section{Results \\ cDNA microarray hybridization}

The htCREB hybridization intensities in the adult testes, fetal testes and spermatozoa samples were 52.36, 22.31 and 14.25 respectively (Fig. 1), indicating a 2.35-fold greater expression in the adult testes than in the fetal testes, with a low expression level in spermatozoa. 


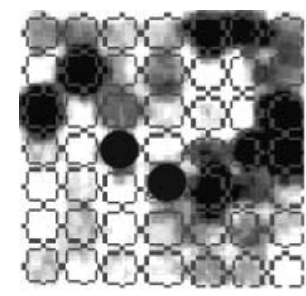

(a) Adult testis

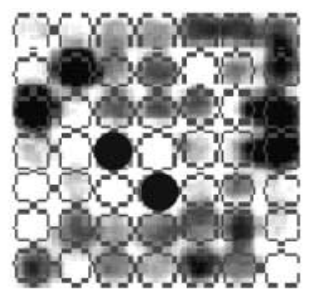

(b) Fetal testis

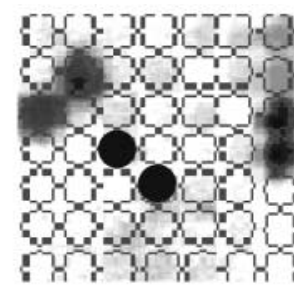

(c) Spermatozoa

Figure 1 Partial cDNA hybridization images showing differential expression of htCREB in adult testis, fetal testis and spermatozoa. The thick black circles indicate htCREB cDNA and the hybridization intensities in adult testis (a), fetal testis (b) and spermatozoa (c), which were 52.36, 22.31 and 14.25 respectively. The hybridization intensity was 2.35 -fold higher in adult than in fetal testis.

\section{Analysis of the cDNA and putative protein sequences}

Sequence analysis revealed that the htCREB transcript was $1650 \mathrm{bp}$ long and consisted of eight exons. The deduced 230-amino acid htCREB protein had the conserved motifs characteristic of the bZIP family members (Fig. 2). BLAST searching of the human genome database (www.ncbi.nlm.nih.gov/genome/seq/HsBlast.html) localized the htCREB gene to human chromosome $2 q 34$, and revealed that it showed high homology to two other fulllength cDNAs (Accession nos. NM_004379, CREB-A; NM_134442, CREB-B) belonging to the CREB family. Further analysis revealed that all three were splice variants transcribed from the CREB gene at $2 q 34$. Splicing comparison of htCREB with the other human CREB variants illustrated that exons $B, C$ and $E$ were identical in the three transcripts, while in htCREB, exon $G$ was spliced out and exons $\mathrm{A}, \mathrm{F}, \mathrm{H}$ and I were shorter than in the other transcripts (Fig. 3). In addition, htCREB exons $\mathrm{A}$ and $\mathrm{H}$ employed alternative $3^{\prime}$ splice site selections, while exons $\mathrm{F}$ and I employed alternative $5^{\prime}$ splice site selections. SMART PROGRAM (Schultz et al. 1998) comparisons of the htCREB (AAQ24858) and other CREB (NP_004370, CREB-A, 327aa and NP_604391, CREB-B, 341aa) aminoacid sequences showed that htCREB lacked the Q2 domain found in the other two transcripts (Fig. 3).

\section{Expression of htCREB transcripts in human testis and sperm}

Our RT-PCR analyses revealed that htCREB transcripts were not detected in fetal testis, but were found in ejaculated sperm, and testes from adult and elderly men, though at relatively lower levels in the testis from elderly men (Fig. 4). Sequence results suggested that this band was the desired PCR product. Furthermore, htCREB was nearly undetectable in the testes of five SCOS patients except for patients 4 and 5 (Fig. 5).

\section{Tissue distribution of htCREB transcripts}

RT-PCR analysis of a multiple tissue cDNA panel revealed that the htCREB mRNA was highly expressed in human testis and weakly expressed in other tissues, such as lung and kidney (Fig. 6). Intriguingly, no expression signal was detected in human ovary, indicating that this alternative splice product is specific to the male.

\section{Discussion}

The CREB proteins are members of a multigene family of transcription factors involved in CAMP-mediated transcription regulation. The factors in this protein family contain the basic transactivation and basic-domain-leucine-zipper (bZIP) DNA-binding domains (Lou \& Gagel 2001), with the transactivation domain divided into two regions: the phosphorylation box (P-box or KID) encoded by exons $\mathrm{E}$ and $\mathrm{F}$, and two glutamine-rich Q1 and Q2 domains, which are chiefly encoded by exons $\mathrm{C}$ and $\mathrm{G}$. Exons $\mathrm{H}$ and I make up the bZIP DNA-binding domains (Fig. 3). The CREB proteins induce target gene expression via their constitutive activation domains (CAD or Q2) and kinaseinducible activation domains (KID), which function synergistically in response to cellular signals (Goto et al. 2002). The KID stimulates transcription via a phospho(Ser133)dependent interaction with the coactivator paralogs, CREB-binding protein and p300, whereas the Q2 domain recruits the TFIID complex via a direct association with hTAFII130 (Asahara et al. 2001, Chaudhary \& Skinner 2001).

The CREB gene contains at least 12 exons, several of which are alternatively spliced to encode a variety of CREB isoforms. Exon D is an alternatively spliced exon of $42 \mathrm{bp}$ encoding 14 amino acids. In this work, the other two CREB isoforms, CREB-A and CREB-B, used for comparison, were produced by exon $\mathrm{D}$ splicing in or out (Fig. 3). Both of these isoforms were widely expressed in many tissues (Berkowitz \& Gilman 1990). In the testis, exons $\mathrm{W}$ (between $\mathrm{G}$ and $\mathrm{H}$ ), $\mathrm{Y}$ (between $\mathrm{D}$ and $\mathrm{E}$ ) and $\mathrm{Z}$ (between $\mathrm{W}$ and $\mathrm{H}$ ) can be alternatively spliced, and all of them are testis-specific and are most strongly detected in germ cells. CREB is predominantly a positive modulator of the cAMP-responsive genes, but in the testis, alternative exon splicing additionally results in the expression of repressor CREB isoforms. When exon $\mathrm{W}$ is spliced into the CREB mRNA, termination of translation by stop codons within exon $\mathrm{W}$ permits in-frame translation to reinitiate at downstream initiation codons, resulting in the production of inhibitor CREB isoforms (I-CREBs). The I-CREBs compete 
GAACGAAAGCAGTGACG GAGGAGCTT GTACCACCGGTAACTAAATGACCATGG AATCTGGA

62

M T M E S G GCCGA GAACCAGCAGAGTGGAGATG CAGCTGTAACAGAAGCTGAAAACCAACA AATGACA

T

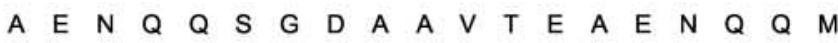
122 GTTCAAGCCCAGCCACAGATT GCCACATT AGCCCAGGTATCTATGCCAGCAGCT CATGCA

A

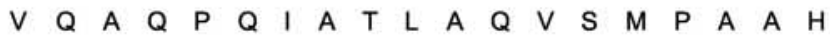
ACATCATCTGCTCCCACCGTAACTCTAGTACAGCTGCCCAATG GGCAGACAGTT CAAGTA

V

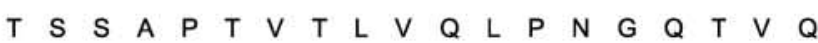
CATGGAGTCATTCAGGCGGCCCAGCCATCAGTTATTCAGTCTCCACAAGTCCAA ACAGTT

V

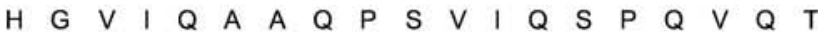
302

CAGTCTTCCTG TAAGGACTTAAAAAGACTT TTCTCCG GAACACAGATTTCAACTA TTGCA 362

Q S S S C K K D L K R R L F S G T $Q$ Q I S GAAAGTGAAGATTCA CAGGAGTCAG TGGATAGTGTAACTGATTCCCAAA AGCGA AGGGAA

E ATTCTITTCAAGGAG GCCTTCCTACAGGAAAATTTTGGATGACTTATCTTCTGATG

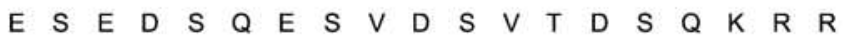
CACCA

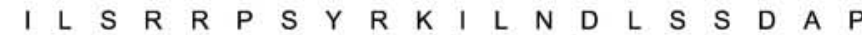
GGAGTGCCAA GGATTGAAGAAG AGAAGTCTGAAGA GGAGACTTCA GCACTTCC TACACAG Q $G \vee P R$ I E E E K S E E E T S A L P T CCTGCTGAAGAAGCAGCACGAAAGAGAGAGGTCCG TCTAATGGAGAACAGGGA AGCAGCT

A

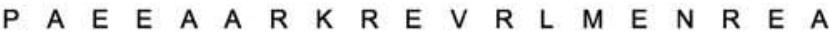
CGAGAGTGTCGTAGAAAGAA GAAAGAATATGTGAAATGTTTAGAAAACAGAGTG GCAGTG $\mathrm{V}$

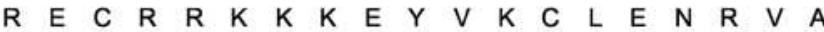
CTTGAAAATCAAAACAAGACA TTGATTGA GGAGCTAAAA GCACTTA AGGACCTTT ACTGC 722

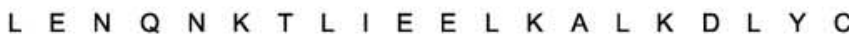
CACAAATCAGA TTAATTTG GGATTTAAATTTTCACCT GTTAAGGTG GAAAATGGA CTGGC 782 H K S D \# TTGGCCACAACCTGA AAGACAAAATAAACAT TTTATTTTCTAAACAT TTCTTTTTTT CTA TGCGCAAAACTGCCTGAAAGCAAC TACAGAATTTCATTCATTTGTGCTTTTGCAT TAAAC 902 TGTGAATGTTC CAACA CCTGCCTCCACTTCTCCCCTCAAGAAAT TTTCAACGCCA GGAAT 962 CAT GAAGA GACTTCTGCTTTTCAA CCC CCACCCTCCTCA AGAAGTAATAATTTGT TTACT 1022 TGTAAATT GATG GGA GAAATGAGGAAAA GAAAATCTT TTTAAAAATG ATTTCAAG GTTTG 1082 TGCTGAG CTCCTTGATTGCCT TAGG GACAGAATTACCCCAG CCTCTTGAGC TGA

Figure 2 (Continued). 


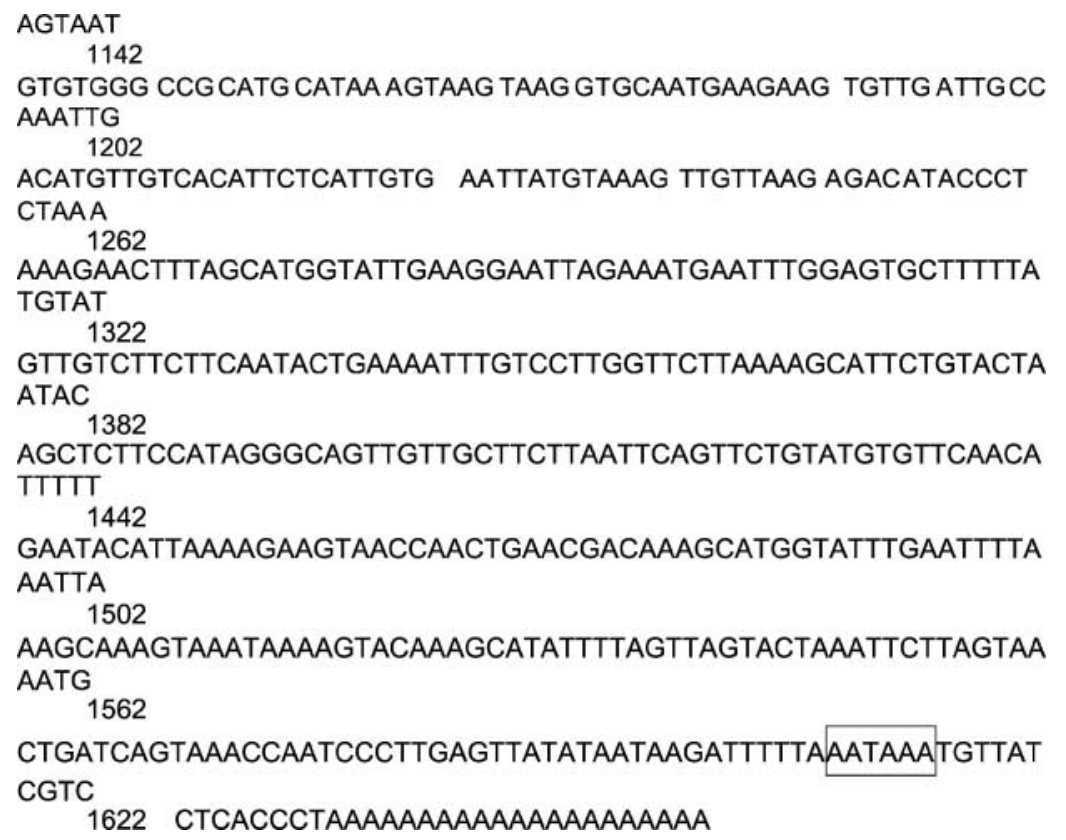

Figure 2 Nucleotide and deduced amino-acid sequence of htCREB. The single-letter code of the predicted amino-acid sequence is indicated below the nucleotide sequence from positions 44 to 736 (230 amino-acid residues). The start and termination codons are shaded. The tail signal is boxed, and the sequences used as the RT-PCR primers are underlined.

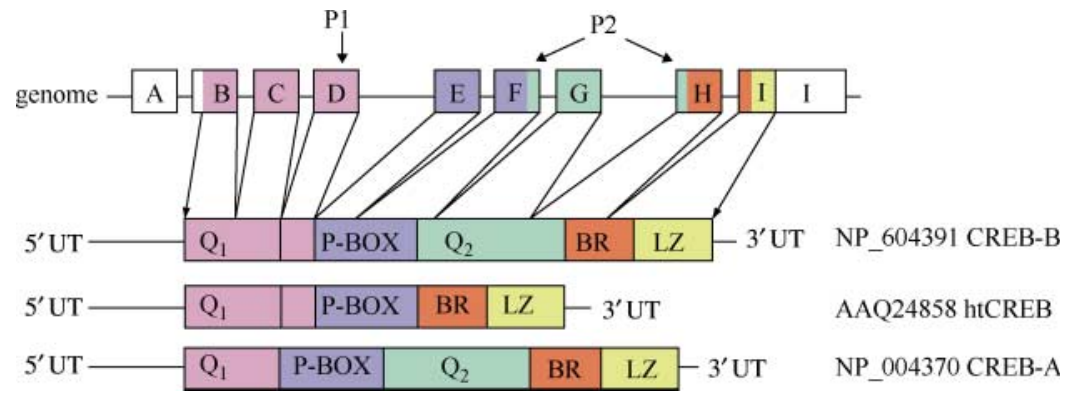

Figure 3 Comparison of amino-acid sequences between htCREB and two other human CREB isoforms: CREB-A and CREB-B. The coding regions, contained between the two arrows, are labeled and boxed, with colors showing corresponding exons and domains. The $\mathrm{Q} 1$ region of CREB-A is shorter than CREB-B due to exon D splicing out. htCREB lacks the Q2 region found in the other spliceoforms (green box). P1: upstream primer, P2: downstream primer. (a)

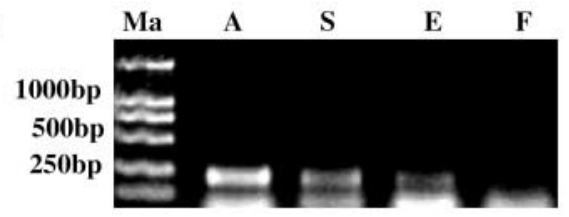

(b)

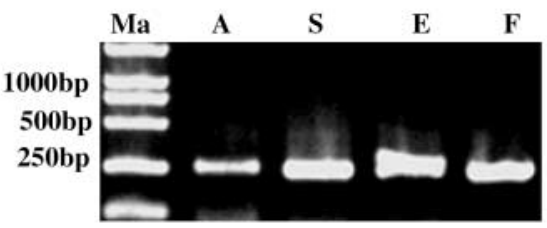

Figure 4 (a) RT-PCR and electrophoresis indicate that htCREB is expressed in adult testis, ejaculated sperm and testis from elderly men, but not in fetal testis. (b) Amplification of $\beta$-actin as the positive loading control. Ma: marker; A: adult testis; $S$ : ejaculated sperm; E: testis from elderly men; F: fetal testis. (a)

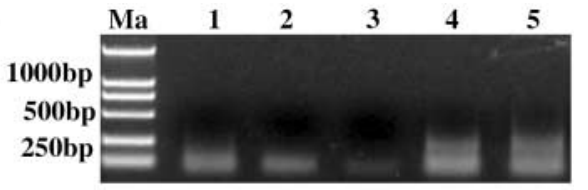

(b)

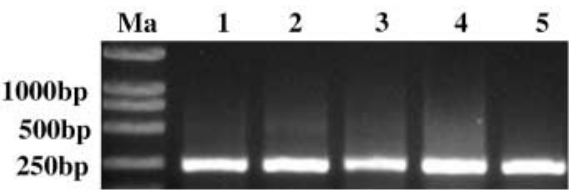



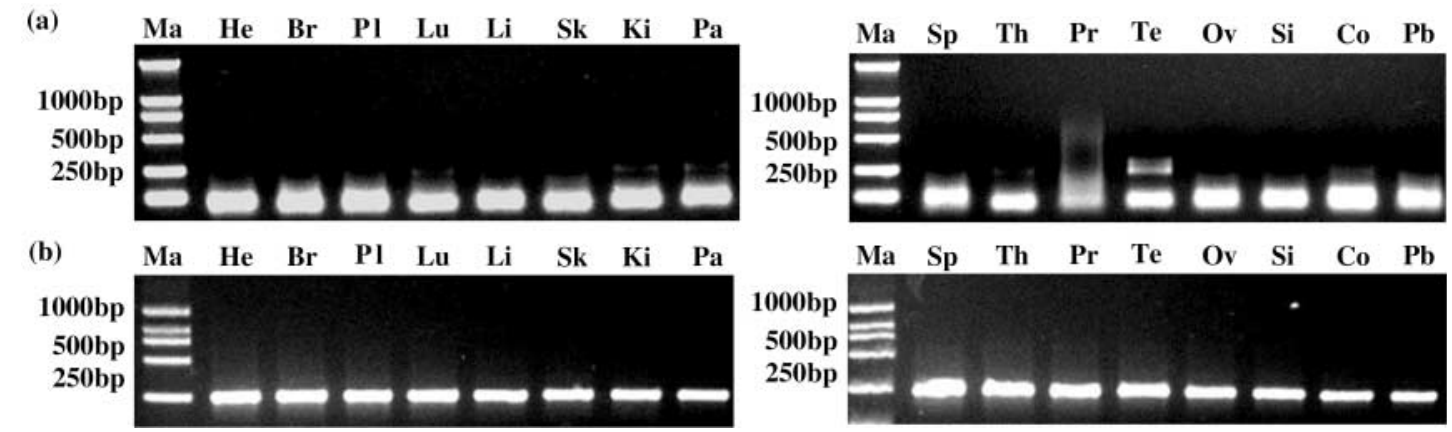

Figure 6 RT-PCR analysis of htCREB tissue distribution in a multiple-tissue panel. (a) Expression of the htCREB transcript (276 bp fragment) was examined in 16 different tissues and found to be highly expressed in testis and weakly expressed in kidney and pancreas. (b) Control amplification of $\beta$-actin. Ma, marker; He, heart; $\mathrm{Br}$, brain; Pl, placenta; Lu, lung; Li, liver; Sk, skeletal muscle, Ki, kidney; Pa, pancreas; Sp, spleen, Th, thymus; $\mathrm{Pr}$, prostate; $\mathrm{Te}$, testis; $\mathrm{Ov}$, ovary; $\mathrm{Si}$, small intestine; $\mathrm{Co}$, colon; $\mathrm{Pb}$, peripheral blood leukocyte.

with CREB for binding to CREs (such as those located in the promoter of the CREB gene) and downregulate CAMPstimulated gene expression. The I-CREBs are expressed at specific stages of spermatogenesis, predominately in spermatocytes, and may account for cell- and stagespecific repression of cAMP-regulated genes (Walker et al. 1996, 1998). Furthermore, insertion of human testisspecific exon $\mathrm{Z}$ after exon $\mathrm{W}$ abolishes the synthesis of one of the two inhibitor CREBs, due to the introduction of an in-frame stop codon within exon $Z$. The splicing in of exon $Z$ may be part of a human-specific mechanism for regulation of cAMP-dependent regulatory pathways in spermatogenesis, by abolishing the expression of a CREB repressor (Girardet et al. 1996).

Here, we used a constructed human adult testis cDNA microarray to identify genes related to human testis development and spermatogenesis, and identified another novel alternative spliceoform of CREB (designated htCREB) in human testis. Hybridization of our human cDNA microarray with adult testis, fetal testis and sperm samples demonstrated that the expression level of the htCREB transcript differed in adult testis from fetal testis by more than twofold, and that the htCREB transcript was expressed in sperm (Fig. 1). Tissue distribution analyses indicated that htCREB was highly expressed in human adult testis but weakly expressed in the other tissues, such as lung and kidney (Fig. 6). Taken together, these results suggest that the htCREB transcript probably plays a role in human germ cell maturation, especially during spermatogenesis.

To further examine whether htCREB was chiefly expressed in germ cells, we first used RT-PCR to examine differential expression at various developmental stages: fetal testis, adult testis, elderly testis and human ejaculated sperm. The htCREB transcript was detected at relatively high levels in adult testis and human ejaculated sperm, and at lower levels in elderly testis, and was not detected in fetal testis (Fig. 4). This result appears to contradict the microarray data, which indicated a positive signal in the fetal testis experiment. As RT-PCR is a sensitive assay, we suggest that the positive microarray result could be due to hybridization of the probe with another, yet unknown, spliceoform of CREB that was not detected with the RT-PCR primers. Developmentally, spermatogenesis does not occur in male embryos. In adults, spermatogenic cells undergo successive mitotic, meiotic and postmeiotic phases, and then form mature sperm. In elderly men, spermatogenesis becomes less efficient, and the quantity and quality of sperm decrease. Thus, we suggest that in the human testis, htCREB may be primarily expressed in germ cells and may function in spermatogenesis. Accordingly, we explored htCREB expression in the testes of five SCOS patients and found that htCREB transcripts were nearly undetectable in such patients (Fig. 5). As SCOS is a condition of the testes in which only Sertoli cells occur in the seminiferous tubules, our results further suggest that htCREB, like the other identified spliceoforms of CREB, is primarily expressed in germ cells.

Next, we focused on examining the putative function(s) of this CREB spliceoform in spermatogenesis via structure and function analyses. Compared with the other spliceoforms CREB-A and CREB-B, htCREB employed both alternative exons and alternative splice site selection. Because exon $G$ is spliced out, the deduced htCREB protein lacks the glutamine-rich Q2 region but contains Q1 and the other CREB domains (Fig. 3). CREB is a bifunctional transcription activator, exerting its effects through a constitutive activation domain (CAD or Q2) and a distinct KID (Felinski et al. 2001). Previous studies of CREB function have yielded conflicting results with regard to its activation of constitutive transcription. Deletion studies performed by Gonzalez et al. (1991) suggested that the $\mathrm{NH}_{2}$-terminal glutamine-rich (Q1) domain of CREB was important for basal activation of the somatostatin promoter in F9 cells. However, these studies did not establish that this domain was capable of providing basal activation alone, and subsequent results indicated that these results might be specific to the experimental model (Quinn 1993). Another study reported that contributions from both the Q1 and Q2 domains were important for basal activation, with Q2 being crucial for basal activity (Quinn 1993). Ferreri and coworkers subsequently showed that deletion of 10 amino acids from the Q2 domain 
significantly reduced the interaction of CREB with hTAFII130, and they concluded that the CREB Q2 domain is fully competent to serve as a strong activator in vitro (Ferreri et al. 1994). Another CREB family member, CREM (cyclic-AMP responsive element modulator), was shown to produce PKA-responsive transcriptional activators, and also exists in isoforms that explicitly antagonize cAMPdependent transcription (Foulkes et al. 1991, Laoide et al. 1993). Exon 9 of CREM (roughly equivalent to exon G of the CREB gene) also codes for a glutamine-rich domain. When exon 9 was inserted into the CREM mRNA by alternate splicing, the CREM protein switched from acting as a repressor to functioning as an activator during spermatogenesis (Foulkes \& Sassone-Corsi 1992). In contrast, CREM proteins lacking Q2 may function as repressors, because they are unlikely to form productive interactions with proteins in the basal transcription complex (Ferreri et al. 1994). Thus, the functionality of htCREB may be considered analogous to that of CREM. With exon G spliced out, htCREB is likely to be a repressor, inhibiting transcription and downregulating cAMP-stimulated gene expression. During spermatogenesis, this novel I-CREB probably competes with the activator CREBs to determine the transcriptional rates of the vitally important cAMPregulated genes (Walker et al. 1996).

In summary, the present study reports the identification of a novel spliceoform of CREB, htCREB, which is chiefly expressed in human male germ cells. With exon $\mathrm{G}$ spliced out, htCREB is likely to be the repressor responsible for downregulating cAMP-dependent gene expression. This identification and analysis of an additional testis-related CREB isoform improves our understanding of gene expression and regulation of spermatogenesis.

\section{Acknowledgements}

The work was supported by grants from National Project 973, China (no. G1999055901), and Chinese Natural Science Funds (no. 30170485).

\section{References}

Andrisani OM 1999 CREB-mediated transcriptional control. Critical Reviews in Eukaryotic Gene Expression 9 19-32.

Asahara H, Santoso B, Guzman E, Du K, Cole PA, Davidson I \& Montminy M 2001 Chromatin-dependent cooperativity between constitutive and inducible activation domains in CREB. Molecular and Cellular Biology $217892-7900$.

Berkowitz LA \& Gilman MZ 1990 Two distinct forms of active transcription factor CREB (CAMP-response element binding protein). PNAS 87 5258-5262.

Chaudhary J \& Skinner MK 2001 Role of the transcriptional coactivator CBP/p300 in linking basic helix-loop-helix and CREB responses for follicle-stimulating hormone-mediated activation of the transferrin promoter in Sertoli cells. Biology of Reproduction 65 568-574.

Cheng LJ, Zhou ZM, Li JM, Zhu H, Zhu H, Zhou YD, Wang LR, Lin M \& Sha JH 2002 Expression of a novel HsMCAK mRNA splice variant, tsMCAK gene in human testis. Life Sciences $\mathbf{7 1}$ 2741-2757.

Daniel PB \& Habener JF 1998 Cyclical alternative exon splicing of transcription factor cyclic adenosine monophosphate response element-binding protein (CREB) messenger ribonucleic acid during rat spermatogenesis. Endocrinology 139 3721-3729.

Felinski EA, Kim J, Lu JF \& Quinn PG 2001 Recruitment of an RNA polymerase II complex is mediated by the constitutive activation domain in CREB, independently of CREB phosphorylation. Molecular and Cellular Biology 21 1001-1010.

Ferreri K, Gill G \& Montminy M 1994 The cAMP-regulated transcription factor CREB interacts with a component of the TFIID complex. PNAS 91 1210-1213.

Foulkes NS \& Sassone-Corsi P 1992 More is better: activators and repressors from the same gene. Cell 68 411-414.

Foulkes NS, Borrelli E \& Sassone-Corsi P 1991 CREM gene: use of alternative DNA-binding domains generates multiple antagonists of cAMP induced transcription. Cell 64 739-749.

Girardet C, Walker WH \& Habener JF 1996 An alternatively spliced polycistronic mRNA encoding cyclic adenosine $3^{\prime}, 5^{\prime}$-monophosphate (CAMP)-responsive transcription factor CREB (CAMP response element-binding protein) in human testis extinguishes expression of an internally translated inhibitor CREB isoform. Molecular Endocrinology 10 879-891.

Gonzalez GA \& Montminy MR 1989 Cyclic AMP stimulates somatostatin gene transcription by phosphorylation of CREB at serine 133 . Cell 59 675-680.

Gonzalez GA, Menzel P, Leonard J, Fischer WH, Montminy MR 1991 Characterization of motifs which are critical for activity of the cyclic AMP-responsive transcription factor CREB. Molecular and Cellular Biology 11 1306-1316.

Goto NK, Zor T, Martinez-Yamout M, Dyson HJ \& Wright PE 2002 Cooperativity in transcription factor binding to the coactivator CREB-binding protein (CBP). The mixed lineage leukemia protein (MLL) activation domain binds to an allosteric site on the KIX domain. Journal of Biological Chemistry 277 43168-43174.

Habener JF, Miller CP \& Vallejo M 1995 Cyclic AMP-dependent regulation of gene transcription by cAMP response element-binding protein and CAMP response element modulator. Vitamins and Hormones 51 1-57.

Hoeffler JP, Meyer TE, Waeber G \& Habener JF 1990 Multiple adenosine $3^{\prime}, 5^{\prime}$-monophosphate response element DNA-binding proteins generated by gene diversification and alternative exon splicing. Molecular Endocrinology 4 920-930.

Huang XY, Wang H, Xu M, Lu L, Xu ZY, Li JM, Zhou ZM \& Sha JH 2004 Expression of a novel RAD23B mRNA splice variant in the human testis. Journal of Andrology 25 363-368.

Laoide BM, Foulkes NS, Schlotter F \& Sassone-Corsi P 1993 The functional versatility of CREM is determined by its modular structure. EMBO Journal 12 1179-1191.

Lou H \& Gagel RF 2001 Alternative ribonucleic acid processing in endocrine systems. Endocrine Reviews 22 205-225.

Meyer TE \& Habener JF 1993 Cyclic AMP response element binding protein CREB and related transcription activating DNA binding proteins. Endocrine Reviews 14 269-290.

Quinn PG 1993 Distinct activation domains within cAMP response element-binding protein (CREB) mediate basal and cAMP-stimulated transcription. Journal of Biological Chemistry 268 16999-17009.

Ruppert S, Cole TJ, Boshart M, Schmid E \& Schutz G 1992 Multiple mRNA isoforms of the transcription activator protein CREB: generation by alternative splicing and specific expression in primary spermatocytes. EMBO Journal 11 1503-1512.

Schultz J, Milpetz F, Bork P \& Ponting CP 1998 SMART, a simple modular architecture research tool: identification of signaling domains. PNAS 95 5857-5864.

Scobey M, Bertera S, Somers J, Watkins S, Zeleznik A \& Walker W 2001 Delivery of a cyclic adenosine $3^{\prime}, 5^{\prime}$-monophosphate response element-binding protein (CREB) mutant to seminiferous tubules results in impaired spermatogenesis. Endocrinology 142 948-954. 
Sha JH, Zhou ZM, Li JM, Yin LL, Yang HM \& Zhou KY 2002 Identification of testis development and spermatogenesis-related genes in human and mouse testis using cDNA microarray. Molecular Human Reproduction 8 511-517.

Waeber G, Meyer TE, LeSieur M, Hermann HL, Gerard N \& Habener JH 1993 Developmental stage-specific expression of cyclic adenosine $3^{\prime}, 5^{\prime}$-monophosphate response element binding protein CREB during spermatogenesis involves alternative exon splicing. Molecular Endocrinology 7 1501-1513.

Walker WH, Girardet C \& Habener JF 1996 Alternative exon splicing controls a translational switch from activator to repressor isoforms of transcription factor CREB during spermatogenesis. Journal of Biological Chemistry 271 20145-20158.

Walker WH, Daniel PB \& Habener JF 1998 Inducible cAMP early repressor ICER down-regulation of CREB gene expression in Sertoli cells. Molecular and Cellular Endocrinology 143 $167-178$.
Wang H, Zhou ZM, Xu M, Li JM, Xiao JH, Xu ZY \& Sha JH 2004 A spermatogenesis-related gene expression profile in human spermatozoa and its potential clinical applications. Journal of Molecular Medicine 82 317-324.

Yin LL, Li JM, Zhu H, Lin M, Cheng LJ, Wang YQ, Zhou ZM \& Sha JH 2002 Identification and characterization of a gene coding a novel isoform of DEAD-box protein. Reproduction, Fertility, and Development 14 185-189.

Zhou ZM, Sha JH, Li JM, Lin M, Zhu H, Zhou YD, Wang LR, Zhu H, Wang YQ \& Zhou KY 2002 Expression of a novel reticulon-like gene in human testis. Reproduction 123 227-234.

Received 8 October 2003

First decision 24 December 2003

Revised manuscript received 19 August 2004

Accepted 20 August 2004 